Resumen Ejecutivo

\section{El Desafío}

Se talan los bosques, las temperaturas suben y se pierde la biodiversidad. Los pobres se vuelven más pobres y las culturas indígenas desaparecen. Con el aumento de la temperatura, aumentan los incendios, se prolongan las sequías, se extienden las inundaciones, y las plagas y enfermedades que atacan al ganado y a las plantas se adaptan y se multiplican. Lo que muchos llaman una "tormenta perfecta" toma fuerza y su impacto se extiende a través del mundo en vías de desarrollo, desde los bosques y granjas hasta la atmósfera. Las primeras y más afectadas son las poblaciones más pobres que viven con dificultad de tierras en las que previamente había bosques, o que cosechan cereales secos en tierras degradadas o de secano, donde no hay margen para errar o es muy pequeño. Luego, son afectadas las zonas de regadío, donde las inundaciones y las sequías se combinan para llenar de cieno o vaciar los embalses; $y$ los agricultores que plantan variedades de cosecha muy específicas luchan por adaptarse. Las ganancias y el sustento de los pueblos más pobres del planeta se desploman.

Esta situación es consecuencia, en gran medida, del mal manejo de nuestros bosques, árboles y recursos
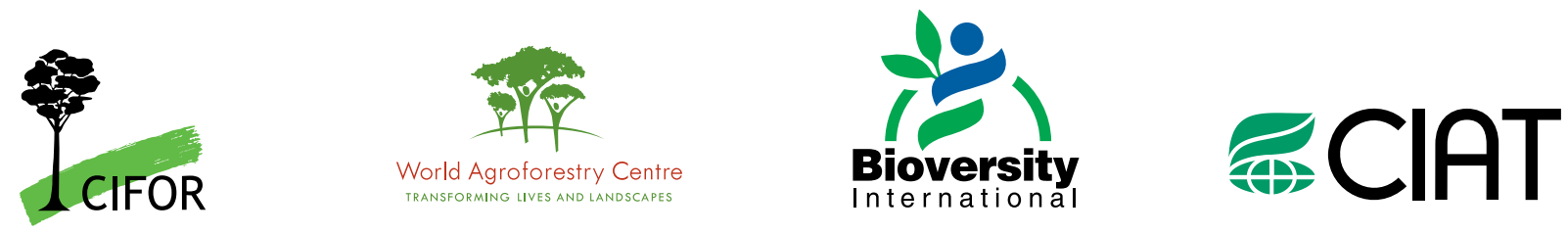


\section{Visión}

Reducción de la pobreza y el hambre, mejora de la salud y la nutrición, y aumento de la capacidad de recuperación del ecosistema mediante investigación agrícola internacional de alta calidad, trabajo en asocio y liderazgo.

\section{Objetivos estratégicos}

- Alimentación para el pueblo: Creación y celeridad en el aumento sostenible de la productividad y la producción de alimentos sanos por y para los pobres.

- Medio ambiente para el pueblo: Conservación, aumento y uso sostenible de los recursos naturales y la biodiversidad para mejorar los medios de vida de los pobres en respuesta al cambio climático y a otros factores.

- Políticas para el pueblo: Promoción de cambios de políticas y cambios institucionales que estimulen el crecimiento agrícola y la equidad para beneficiar a los pobres, especialmente a las mujeres en zonas rurales y a otros grupos desfavorecidos.

Fuente: CGIAR. 2010. A strategy and results framework for the CGIAR. http://www.cgiar.org/changemanagement/pdf/cgiar_srf_june7_2010.pdf (5 September 2010).

genéticos silvestres. A pesar de las décadas de esfuerzos de investigación y desarrollo para revertir la deforestación, la degradación de los bosques y la pérdida de la diversidad biológica, estas tendencias continúan a un ritmo alarmante. Durante el tiempo que toma leer este documento, tanto como 3000 hectáreas de bosques naturales y cobertura arbórea van a desaparecer junto con la biodiversidad que ellos contienen, una pérdida de casi 13 millones de hectáreas anuales. La deforestación y el cambio del uso de la tierra contribuyen un $12-18 \%$ del total anual mundial de las emisiones de carbono, acelerando así el calentamiento global.

Los bosques naturales son parte de un área de tierra finita cada vez más reducida, donde la conversión a la agricultura representa la amenaza más importante en las zonas tropicales en vías de desarrollo. Las tierras de cultivo adyacentes o recientemente cultivadas pueden conservar árboles remanentes o pueden permitir la regeneración natural de los mismos. Sin embargo, estos no son suficientes para brindar los bienes y servicios medioambientales que antes provenían de los bosques intactos. Y aunque la conversión de los bosques a la agricultura puede, en algunos casos, mejorar los ingresos rurales, la mayoría de las veces la deforestación lleva al empobrecimiento, tanto del ecosistema como de las comunidades.

Tales resultados son casi enteramente la consecuencia de fracasos en la gobernanza a nivel del paisaje, y a escala nacional y mundial. Esos fracasos en la gobernanza se manifiestan típicamente a través de factores tales como la falta de claridad en la tenencia de la tierra, o derechos inseguros de acceso a los recursos; regímenes de extracción y de comercio e inversión mal reglamentados; planificación inexistente o incompleta del uso de la tierra; un aumento en la tendencia a la apropiacion ilegal de tierras; incentivos adversos; exclusión de la toma de decisiones a pueblos pobres, por lo general indígenas; $y$ una inadecuada aplicación de la ley. En forma individual o colectiva, estos factores contribuyen a la pérdida de bosques y cobertura arbórea , al agotamiento progresivo de los recursos genéticos arbóreos y de la biodiversidad, y a la distribución desigual de los beneficios económicos y sociales provenientes de de los bosques, árboles y de los sistemas agroforestales.

La deforestación y la degradación ocasionan más que sólo la pérdida de biodiversidad, productos y servicios medioambientales provistos por los bosques y los árboles como captación de carbono, estabilización de los suelos, adaptación a los efectos destructivos del aumento de las temperaturas o un simple y calmo refugio. La falta de optimización del uso de la tierra significa que estamos desperdiciando una oportunidad para mejorar tanto el sustento de más de mil millones de los habitantes más pobres del planeta, asi como los estados financieros de los países en vías de desarrollo. Sólo en Indonesia, se pierden más de US\$ 3000 millones al año debido a la tala ilegal. Los bosques y árboles proveen, en cifras conservadoras, US\$ 250,000 millones en varias formas de ingresos de recursos como madera, madera para combustible, alimentos, medicinas y productos forestales no madereros. Sin embargo, esta cifra podría ser mayor y podría también ser sustentable para las generaciones venideras.

Los estudios muestran que las poblaciones que viven en o cerca de los bosques obtienen un promedio de cerca del $25 \%$ de sus ingresos de los recursos forestales; esta cifra podría ser mucho más alta si se aplicaran los enfoques de manejo de uso múltiple que tienen como objetivo la obtención de todas las fuentes potenciales de ingresos de los bosques, árboles y servicios medioambientales, en vez de la extracción limitada a unas pocas especies valiosas de árboles. El potencial de pagos por servicios medioambientales (PSA), y específicamente la reducción de la emisiones provenientes de la deforestación y la degradación (REDD+, por sus siglas en inglés), como 
fuentes de ingresos para administradores forestales rurales permanecen apenas aprovechadas. Los árboles que están junto con tierras de cultivo ofrecen un gran potencial para aumentar los ingresos rurales. Aproximadamente el 10\% de la cubierta mundial forestal se encuentra asociado a tierras de cultivo -y la tendencia va en aumento-, contribuyendo así en forma importante a la mitigación y adaptación climática. En los países en vías de desarrollo, los sistemas agroforestales proporcionan forraje $y$ productos forestales no madereros escenciales, y contribuyen en forma significativa a los ingresos en hogares encabezados por mujeres. Las especies de árboles silvestres tienen el potencial de jugar un rol crítico en la mejora de los ingresos en las fincas pequeñas. Sin embargo, la mayoría de los agentes de extensión no reciben entrenamiento en técnicas agroforestales y la mayoría de las especies de árboles silvestres todavía no se conserva de un modo adecuado. Si se entrenase debidamente a los agentes de extensión, si las especies de árboles silvestres fuesen clasificadas y cultivadas con el fin de preservar y mejorar su productividad sustentable, y si el acceso a mercados para productos forestales fuese mejorado, entonces, el ingreso proveniente de los árboles en las fincas agrícolas podría ser aumentado enormemente.

El mundo requiere un esfuerzo a largo plazo, bien planificado y con grandes recursos para mejorar el manejo y gobernanza de los bosques que aún quedan, para reducir los conflictos por tierras en disputa, para aumentar la participacion de mujeres y de comunidades marginalizadas y para obtener más ganancias de los árboles deliberadamente cultivados en tierras agrícolas y en tierras adyacentes a bosques. Ante la ausencia de semejante esfuerzo, aquellas poblaciones que dependen de los bosques y árboles para su sustento se empobrecerán aún más, y el cambio climático continuará calentando el planeta.

Lo que se necesita ahora para responder a este desafío es un nuevo enfoque de investigación más estratégico, más enfocado y más colaborativo. Debe ser ambicioso y de mayor alcance. Debe ser conducido por innovación, por nuevos métodos, por nuevas alianzas y por mayor capacidad. El tiempo que lleva desde la implementación de la ciencia hasta el impacto debe ser reducido. El paso del tiempo no es un aliado de los bosques ni de los árboles.

\section{Un nuevo enfoque de investigación}

En respuesta a la urgencia del desafío arriba indicado, cuatro centros dentro del Grupo Consultivo para la Investigación Agrícola Internacional proponen el Programa de Investigación $N^{\circ} 6$ del CGIAR: Bosques, Árboles y Agroforestería: Medios de Subsistencia, Paisajes y Gobernanza (CRP6 de sus siglas en inglés). Esta iniciativa une a cuatro de los centros de investigación líderes del mundo en sus temas específicos - Centro Mundial de Agroforestería, CIFOR, CIAT y Bioversity -, junto con sus asociados, información, recursos y experiencia, y los enfoca hacia un claro objetivo: ampliar el manejo y uso de los bosques, la agro silvicultura y los recursos genéticos forestales desde el paisaje de bosques hasta fincas agrícolas.

El CRP6 está diseñado para hacer una contribución importante a la visión y los objetivos estratégicos mencionados en el Marco de los Resultados Estratégicos de CGIAR (véase recuadro pág. 4) mediante:

1. La ampliación de la contribución de los bosques, la a agroforestería y los árboles en la producción e ingresos de las comunidades y de los minifundistas que dependen de los bosques;

2. La conservación de la diversidad biológica, incluyendo la diversidad genética de árboles, a través del manejo sustentable y la conservación de los bosques y árboles;

3. El mantenimiento o ampliación de los servicios medio ambientales de los bosques, la agroforestería y los árboles en paisajes multi funcionales y dinámicos;

4. La reducción de emisiones de gases de efecto invernadero y el aumento de las reservas de carbono por medio de un mejor manejo de fuentes forestales y arbóreas junto con el incremento de la resiliencia local y de la sociedad mediante medidas de adaptación forestales, agroforestales y de árboles; y

5. La promoción de los impactos positivos y la reducción de los impactos negativos del comercio global y la inversión como impulsores del cambio paisajístico que afecta a las tierras forestales, las zonas de agro silvicultura, los árboles y al bienestar de los pueblos locales.

La innovación es fundamental para el CRP6, desde el diseño hasta la ejecución, desde el modo en que elegimos a nuestros socios hasta la forma en que nos comunicamos. El CRP6 representa enfoques de avanzada que incorporan la investigación global comparativa con un horizonte temporal extendido (en ambos sentidos, hacia el pasado y hacia el futuro, para poder entender mejor las tendencias), que abarca escalas, sistemas ecológicos, paisajes, instituciones, sectores de la sociedad y disciplinas. Desarrollaremos herramientas complejas, enfoques y marcos para respaldar nuestra investigación, para probar intervenciones y para evaluar y definir opciones políticas y situaciones. Compartiremos aún más nuestro conocimiento e información para lograr un alto impacto. La implementación de los enfoques innovadores del CRP6 y sus impactos llevará a los centros colaboradores del CRP6 y a sus socios principales más allá de las prácticas habituales abriendo nuevas oportunidades para la integración y la sinergia entre los centros y con otros socios, dentro de un marco geográfico, medio ambiental y social más amplio relacionado a los bosques y árboles. 


\section{El marco}

Los bosques existen bajo variados regímenes geográficos, edafológicos y climáticos, y se extienden desde las regiones boreales hasta los trópicos; se estima que casi 560 (68\%) de las eco regiones terrestres en todo el mundo pueden ser bosques y masas forestales. Sin embargo, los árboles no están limitados a dichos hábitats; constituyen un elemento importante en muchos otros sistemas, incluyendo los paisajes agrícolas, pastizales, estepas y desiertos. Esta diversidad ecológica, junto con las grandes variaciones culturales y socio-económicas de los pueblos que viven en o cerca de los bosques y que, de algún modo, dependen de éstos y de la agroforestería, hace que su manejo y uso sea complejo y que requiera una diversidad amplia de estrategias de investigación.

Al mismo tiempo, sabemos que, históricamente, países con bosques han pasado por periodos de disminución y luego, aumento de sus áreas forestadas, con cambios tanto en el tipo como en la extensión de la cobertura forestal en paisajes, según se ilustra a continuación. El progreso de un país o región a lo largo de esta curva de transición del uso del bosque y la tierra ha tendido a seguir el cambio demográfico y el desarrollo económico. Sin embargo, esta curva también es útil para describir la variación espacial a través de los paisajes contemporáneos. Según se ilustra a en la siguiente figura, todos los componentes de investigación del CRP6 se ocupan de los desafíos del manejo del uso de la tierra a través del rango de variación. Por lo tanto, el CRP6 está ideado para llevar a cabo investigación a todo lo largo del continuo del paisaje, desde el bosque relativamente prístino hasta la tierra agrícola de explotación intensiva. El continuo brinda un concepto útil integrador y analítico, dado que las estrategias y enfoques pueden variar de un modo consistente a través del paisaje.

La investigación del CRP6 se centrará en zonas donde los pueblos locales dependen de recursos del bosque y de la agroforestería para su sustento, donde los bosques que son importantes para el secuestro del carbono u otros servicios medio ambientales se encuentran bajo una seria presión debido a extracción de madera o a su conversión en otros usos de la tierra, y/o donde se proyecta que los bosques estarán seriamente afectados por el cambio climático.

Una variedad de impulsores afecta el ritmo de cambio a lo largo de la curva, y cuánto éstos afectan a los servicios medio-ambientales y a los beneficios o déficits de los medios de vida depende principalmente del modo en que están administrados.

Otra novedad será el enfoque de muchas de las investigaciones del CRP6 en los "paisajes centinelas". Dicha investigación respaldará la compilación de datos a largo plazo, lo cual es necesario para entender los impulsos e impactos del cambio del uso de la tierra. Los paisajes

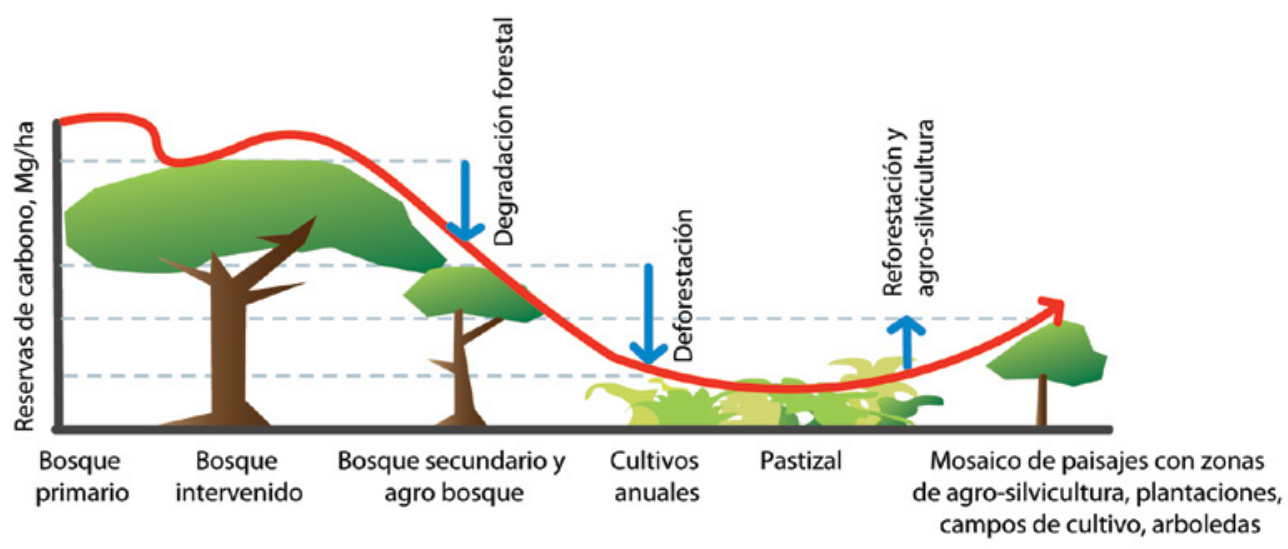

C1 $\begin{aligned} & \text { Sistemas y mercados } \\ & \text { de producción minifundista }\end{aligned}$

C2 Manejo y conservación de los

C3 Manejo del paisaje para los servicios biodiversidad y medios de vida

C4 Adaptación y mitigación del cambio climático

C5 Impacto del comercio y la inversión

C5 en los bosques y pueblos

Componentes del CRP6 dentro de la curva de transición del uso del bosque y la tierra 
centinelas también ofrecerán una ubicación excelente para fomentar el diálogo entre las distintas partes interesadas y para probar modelos, facilitando así el consenso en temas polémicos, como la explotación sostenible de un recurso natural en disputa. También ofrecerán oportunidades para implementar diseños experimentales para medir la adopción de resultados de investigación y para la evaluación de impacto general. Por último, los paisajes centinelas proporcionarán puntos centrales globales para la investigación multidisciplinaria; también proveerán espacios para el encuentro de un grupo más amplio de investigadores, esfuerzos de desarrollo y partes interesadas que trabajan en áreas rurales, incluyendo otros esfuerzos de investigación de largo plazo, específicos a un lugar, que son abordados dentro de la red más amplia del CGIAR.

\section{Catálogo de investigación}

Después de haber consultado de manera extensiva con socios y partes interesadas, hemos identificado cinco componentes que formarán el núcleo de la investigación del CRP6 y las estrategias de impacto. Los cinco componentes del CRP6 están diseñados para proporcionar resultados distintos pero interconectados a través de la curva de transición del uso del bosque y de la tierra, y juntos generarán un conjunto común de impactos. Mediante caminos de impacto cuidadosamente articulados, nuestra investigación estará orientada a producir resultados e impactos medibles y significativos, a nivel global, regional y local. Un resumen de cada componente se detalla a continuación.

1. Sistemas y mercados de producción minifundista. Temas fundamentales de investigación:

- Aumento de productividad y sustentabilidad de las prácticas de forestería y agroforestería del minifundista, incluyendo seguridad alimentaria y beneficios nutritivos, mediante la mejor gestión de sistemas de producción.

- Aumento en la generación de ingresos e integración de mercado para los minifundistas mediante el uso de opciones forestales y de agroforestería.

- Mejora de políticas e instituciones para incrementar los bienes sociales y para asegurar los derechos a los bosques, los árboles y a la tierra.

2. Manejo y conservación de recursos forestales y arbóreos. Temas fundamentales de investigación:

- Conocimiento de las amenazas contra las poblaciones de especies importantes de árboles y formulación de estrategias de conservación genética efectivas, eficientes y equitativas.

- Conservación y caracterización del germoplasma de alta calidad perteneciente a especies de árboles de alto valor a lo largo del gradiente del bosque a la finca.
- Desarrollo de prácticas mejoradas de forestería y control para el manejo de uso múltiple de los ecosistemas forestales.

- Desarrollo de herramientas y métodos para resolver conflictos relativos a la distribución de beneficios $y$ derechos en el uso de recursos forestales y arbóreos.

3. Manejo del paisaje para servicios medioambientales, conservación de la biodiversidad y medios de vida.

Temas fundamentales de investigación:

- Entendimiento de los generadores de la transición forestal como requisito para su gestión.

- Entendimiento de las consecuencias de la transición del bosque hacia bienes y servicios ambientales y medios de vida.

- Mejoramiento de las opciones de respuestas y políticas para sustentar y maximizar los beneficios medioambientales y sociales de los paisajes multifuncionales.

4. Adaptación y mitigación del cambio climático. Temas fundamentales de investigación:

- Utilización de bosques, árboles y agroforestería para la mitigación del cambio climático.

- Mejoramiento de la adaptación al cambio climático mediante bosques, árboles y agroforestería.

- Entendimiento del rol que juegan los bosques, árboles y la agroforestería para alcanzar sinergias entre la mitigación y la adaptación al cambio climático.

5. Impactos del comercio y la inversión en los bosques y en los pueblos. Temas fundamentales de investigación:

- Entendimiento de los procesos e impactos del comercio y la inversión relacionados con el bosque.

- Mejoramiento de las opciones de respuestas y políticas para mitigar los impactos negativos e incrementar los impactos positivos del comercio y la inversión.

\section{Caminos hacia el impacto}

El CRP6 incorporará sus actividades principales de investigación en caminos de impacto específicos para cada componente, y explicará cómo las informaciones obtenidas de la investigación llevarán a resultados e impactos finales. La investigación tendrá como resultado un aumento de conciencia y entendimiento de los problemas y oportunidades entre las principales partes interesadas y quienes determinan las políticas, para mejorar así las técnicas y desarrollar políticas y mecanismos de gobernanza más apropiados y efectivos, y que resulten en verdaderos impactos aplicables a un mundo real. 


\section{Temas}

Componente 1
- Mejoramiento de productividad y
sustentabilidad en las prácticas de silvicultur
y agroforesteria de minifundistas, incluyend
la seguridad alimentaria y los beneficios
nutritivos mediante una mejor gestión de los
sistemas de producción.
- Aumento de la generación de ingresos y la
integración de mercado en el uso por parte
de minifundistas de las opciones de
silvicultura y de agroforestería.
- Mejora de politicas e instituciones para
incrementar los bienes sociales y asegurar los
derechos a los bosques, arboles y a la tierra.
Componente 2
- Conocimiento de las amenazas contra las
poblaciones de importantes especies
arbóreas y formulación de estrategias de
conservación genética efectivas, eficientes
y equitativas.
- Conservación y caracterización del
germoplasma de alta calidad de especies
arbóreas de alto valor en el gradiente del
bosque a la finca.
- Desarrollo de prácticas mejoradas de
silvicultura y control, para el manejo de
uso múltiple de los ecosistemas forestales.
Desarrollo de herramientas y métodos
para resolver conflictos relativos a la
distribución de beneficios y derechos en el
uso de los recursos forestales y arbóreos.

\section{Componente 3}

- Entendimiento de los generadores de la transición forestal como un requisito para su gestión.

- Entendimiento de las consecuencias de la transición forestal para los bienes y servicios medioambientales y para los medios de vida.

- Mejoramiento de las opciones de respuestas y politicas para sustentary maximizar los beneficios medioambien tales y sociales de los paisajes multifuncionales.

\section{Componente 4}

- Utilización de bosques, árboles y agroforestería para la mitigación del cambio climático.

- Mejoramiento de la adaptación al cambio climático mediante bosques, árboles y agroforesteria.

- Entendimiento del rol que juegan los bosques, árboles y la agroforesteria para lograr sinergias entre la mitigación y adaptación al cambio climático.

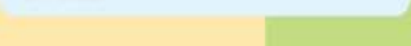

\section{Componente 5}

- Entendimiento de los procesose impactos del comercio y la inversión forestal.

- Ampliación de las opciones de

respuestas y politicas para mitigar los impactos negativos e incrementar los impactos positivos del comercio y la inversión.

\section{Resultados}

Impacto

vulnerables con un mejor acceso y mejores derechos de gestión de los recursos forestales, agro forestales y arbóreos.

Estrategias de impacto

Creación conjunta de estrategias de impacto con socios.

Enfoques de investigación que involucren desde el comienzo a las comunidades de políticos y de profesionales.

Métodos de investigación que incorporen la valoración de impacto $y$ las dimensiones de género.

Inversión estratégica en la evaluación y consolidación de capacidad.

Uso de opciones de medios de comunicación tradicionales $y$ nuevos para el intercambio de conocimiento y comunicaciones.
- Conocimiento del manejo de árboles y acceso al

germoplasma arbóreo productivo al alcance de las comunidades rurales.

Más fácil acceso a los mercados forestales y de productos arbóreos mediante cadenas de valor mejoradas.

- Aumento del conocimiento del estatus y las amenazas a las especies arbóreas prioritarias a nivel local, regional e internacional.

- Implementación de estrategias de conservación efectivas y equitativas en un mayor número de paises.

cunservacios. - Conservacion del material genético arboreo mejorado para su uso por parte de administradores forestales $y$ agricultores.

- Mejora en el manejo de los bosques.

- Uso de la tierra y planeamiento de desarrollo más realista y reforma institucional de las normas que rigen el uso de la tierra.

- Aclaración de derechos, definición de los múltiples interesados a dirigirse, negociaciones con las dirigirse, negociaciones con las
múltiples partes interesadas, e múltiples partes interesadas, inversiones de recuperación.

- Fortalecimiento de acciones realistas, condicionales y voluntarias en una perspectiva de aprendizaje continua.

- Integración adecuada de las inquietudes de REDD + y de EBA dentro de acuerdos globales. Diseño e implementación de - políticas e iniciativas de mitigación y adaptación (M\&A) efectivas, eficientes y equitativas.

- Aumento del apoyo a las iniciativas forestales y arbóreas por parte de mecanismos financieros vinculados al cambio climático.

- Mejor reglamentación del flujo del comercio e inversiones globales para ser responsables en el ámbito para ser socialy ecologico y para prom Fortalecimiento de administrador forestales y de consumidores forestales y de consumidores
globales en la toma de decisiones globales en la toma de decisiones

responsab
forestal.

- Adopción por parte del comercio mundial de pautas para reducir las inversiones nocivas para el ámbito ecológico y social.
Impacto del Programa de Investigación de CGIAR (CRP, por sus siglas en inglés)

- Reducción de la deforestación y la degradación.

- Aumento en las reservas de carbono netas.

- Conservación y aumento de uso de recursos genéticos forestales y arbóreos. - Aumento de los beneficios sociales y económicos de los bienes y servicios forestales y agro-forestales.

- Disminución en el riesgo de los modos de vida rurales.

- Incremento en el acceso de mujeres y otros grupos desfavorecidos a todo tipo de beneficios.

Visión del Marco de

Resultados Estratégicos

(SRF, por sus siglas en

$$
\text { inglés) }
$$

- Creación y mayor rapidez en los aumentos sostenibles de la productividady producción de alimentos saludables por y para los pobres;

- Conservación, incremento y uso sostenible de los recursos naturales y la biodiversidad para mejorar los modos de vida de los pobres como respuesta al cambio climático y a otros factores; $y$

- Promoción de cambios políticos e institucionales que estimulen el crecimiento agricola y la equidad para beneficiar a los pobres, especialmente a mujeres en el ámbito rural y a otros grupos desfavorecidos. 
Los cinco componentes están fuertemente interrelacionados e interconectados, y trabajarán juntos en sinergia para lograr así impactos, según se detalla en la figura a continuación.

\section{Compromisos de amplio alcance}

El diseño del CRP6 también incluye una serie de compromisos para llevar a cabo investigaciones para el desarrollo de modo que produzcan resultados más efectivos y equitativos.

\section{Sensibilidad hacia el género}

Por décadas, el análisis de género se trató de un modo superficial en la investigación agrícola y forestal. Además, silvicultores y extensionistas, administradores de proyectos, políticos e investigadores, siempre han omitido el género en sus trabajos. Esto sucede a pesar de que numerosos estudios han demostrado que el aumento de la participación femenina mejora el manejo de los recursos, ya sea en la comunidad, el hogar o en el agro, así como también produce incrementos en los medios de subsistencia. El género está integrado en todos los componentes y actividades del CRP6. Los métodos de análisis de género producirán el entendimiento de importantes contextos institucionales, culturales y de conductas que establecen la desigualdad y desperdician oportunidades para mejorar la vida de las mujeres. Nuestro enfoque incluirá una compilación de datos separada por sexo, desarrollo de asociaciones y alianzas centradas en género, intercambio de conocimiento y aprendizaje adaptable. La investigación del CRP6 identificará también políticas, tecnologías y prácticas que mejorarán la igualdad de género para el acceso, uso y manejo de bosques y árboles, y la distribución de los beneficios asociados.

A la vez que destacamos el género, el CRP6 también dará prioridad a otros grupos desfavorecidos, como poblaciones indígenas, los jóvenes y los ancianos.

\section{Consolidación de la capacidad}

La mayoría de los países en vías de desarrollo en los que actuará el CRP6 tienen grandes vacíos en cuanto a capacidad. Hay muy pocos ingenieros forestales y especialistas en agroforestería debidamente entrenados, y aún menos expertos multidisciplinarios que abarquen las ciencias biofísicas, sociales, económicas y políticas. Además, el problema está empeorando con una marcada reducción en el entrenamiento y educación en ciencias forestales. Hay menos estudiantes inscribiéndose en esta carrera y las universidades están cerrando sus escuelas de ciencias forestales a un ritmo preocupante.
El refuerzo de la capacidad no es opcional para el CRP6, es más bien un ingrediente fundamental de cómo el impacto del proyecto se orientará. La investigación documentará y aumentará el entendimiento de las necesidades de capacidad mundial indispensables para el manejo y conservación de los bosques, la silvicultura y los recursos genéticos arbóreos. El aumento en la percepción actual sobre la importancia global de los problemas forestales brinda una rara oportunidad para formar una nueva generación de profesionales capaces de asumir la gama de desafíos y oportunidades que los bosques, árboles y la agroforestería proporcionan. Reconocemos la necesidad de contar con conocimientos más complejos, -multi y transdisciplinarios-, de tener un mayor número de trabajadores entrenados dentro de las disciplinas y de que hayan instituciones de mayor capacidad. Aunque la creación de la capacidad al nivel necesario va más allá del ámbito del CRP6, la aumentaremos y activaremos a través de aprendizaje en conjunto e implementación con socios nuevos y con los ya existentes.

\section{Asociaciones}

Un tercer compromiso, junto con nuestros enfoques de género y creación de capacidad, es el modo en que abordamos las asociaciones, constituyendo el camino más importante hacia el impacto.

Convocaremos como asociados de investigación, a los expertos líderes en el mundo mediante asociaciones nuevas o ya existentes, con institutos de investigación especializados y nacionales, y con otras organizaciones de investigación específicas, necesarias para complementar las capacidades fundamentales del CGIAR. También trabajaremos con "socios del ámbito político y profesional” en calidad de clientes inmediatos de nuestros resultados de investigación. Los socios del ámbito profesional y político abarcarán desde foros de negociación global hasta organizaciones comunitarias locales. Además, estableceremos relaciones laborales con "socios para el intercambio de información” para actuar como intermediarios con los medios de comunicación, estudiantes y el público general. En línea con nuestro enfoque de caminos de impacto, trabajaremos estrechamente con asociados a nivel nacional y local, para evaluar y crear capacidad, tanto para llevar a cabo las investigaciones como para tomar decisiones sobre las mismas, para asegurar que resultados e impactos medibles y significativos ocurran en forma global, regional y local.

\section{Intercambio de conocimiento}

Parte de la razón por la cual las ciencias forestales y la agro silvicultura no se han transformado más amplia y 
rápidamente en cambios de política y práctica ha sido debido a la mala comunicación. El modelo de intercambio de conocimiento presentado en este CRP combina los resultados de investigación tradicionales y el alcance de los medios de comunicación con una propagación rápida y multidireccional y un sistema de retroalimentación. Este intercambio influenciará en las herramientas de medios de comunicación social existentes y emergentes, "comunidades miembro", conceptos, tendencias y técnicas de control nuevas. Está diseñado para asegurar que todos los resultados de la investigación, incluyendo los datos mismos, lleguen hoy, y no dentro de cinco años, a los individuos que los necesitan: científicos, profesionales, donantes, agencias de desarrollo, diseñadores de políticas, medios de comunicación y ONG. El CRP6 liderará el camino para desarrollar el intercambio de información como parte integral de la investigación agrícola.

\section{Administración}

El CIFOR, el Centro Mundial de Agroforestería, el CIAT y Bioversity liderarán la implementación del CRP6. Las estructuras de administración, que pretenden ser simples, incluirán un Centro Líder encargado de la responsabilidad fiduciaria y legal para el CRP6. Un Comité Directivo compuesto por los principales centros participantes en el CGIAR, más otras instituciones del CGIAR e instituciones externas asociadas, según sea necesario para la implementación de este ambicioso programa, proporcionará dirección y supervisión. Un Comité de Asesoría Científica y de Participantes Interesados proveerá pautas para asegurar relevancia, y una Unidad de Apoyo de Gestión basada en el Centro Líder se encargará de la administración y coordinaciones diarias. Equipos de Implementación de Componentes, integrados por científicos de distintos centros participantes y organizaciones asociadas, estarán encargados de llevar a cabo la investigación y otras actividades necesarias para lograr los rendimientos y resultados del CRP6, y obtener, en última instancia, los impactos deseados.
El CRP6 establecerá mecanismos para asegurar la calidad, relevancia e impacto de nuestras investigaciones, $y$ desarrollará procedimientos para el control y la evaluación de actividades, proyectos y procesos. La administración del CRP6 se centrará en la promoción de excelencia científica y de una gestión adaptable, caracterizada por la transparencia, justicia e integración.

\section{Recursos necesarios}

Para lograr estos ambiciosos objetivos a través de este programa, el CRP6 necesitará un presupuesto inicial de 232.9 millones de dólares estadounidenses para los próximos tres años, por lo que sería un programa de 10 años como mínimo. En el primer año, se prevé un presupuesto de US\$ 67.8 millones, de los que se espera US\$ 23 millones de financiación no restringida, US\$ 33.5 millones de fondos restringidos de proyectos confirmados, y los restantes US\$ 11.3 millones de propuestas aún no confirmadas. Los fondos restringidos incluyen aquellos en curso. Esto representa solo un aumento pequeño de las prácticas habituales ya que los centros participantes alinean sus programas de investigación con el CRP6 y establecen capacidad de administración y comunicaciones a nivel de Consorcio. En el segundo y tercer año, proyectamos mayor nivel de financiación, para permitirnos así implementar los aspectos más innovadores de la propuesta, como por ejemplo, la red de paisajes centinelas. Anticipamos que, en los años venideros, este nivel de financiación impulsará otras grandes inversiones para investigación, tanto por los asociados del CRP6 como por los miembros externos. Además, se necesitará considerable financiación para complementar la creación de capacidad forestal y la implementación de agendas, más allá del alcance de esta iniciativa de investigación para el desarrollo; estas agendas serán necesarias para que los caminos de impacto puedan obtener los resultados al nivel esperado.

La propuesta del CRP6 está disponible en www.cifor.cigiar.org/crp6/ con carácter provisional hasta que se establezca la estructura de comunicaciones del CRP6. Para más información, póngase en contacto con nosotros en cgiarforestsandtrees@cgiar.org. del Grupo Consultivo sobre Investigación Agrícola Internacional. El programa tiene como objetivo mejorar la gestión y el uso de los bosques, la agroforestería y los recursos genéticos de árboles a través del paisaje desde bosques hasta fincas. El Programa de colaboración está dirigido por el Centro de Investigación Forestal Internacional en colaboración con el Centro Mundial de Agroforestería, Bioversity y el Centro Internacional de Agricultura Tropical. 